\title{
To stress or not to stress: a new approach for diagnosing coronary heart disease
}

\author{
Kaitlyn Mikielski, Matthew Budoff \\ Department of Internal Medicine, Lundquist Institute, Los Angeles Biomedical Research Institute at Harbor-UCLA, CDCRC, Torrance, CA, USA \\ Correspondence to: Matthew Budoff. Department of Internal Medicine, Lundquist Institute, Los Angeles Biomedical Research Institute at Harbor- \\ UCLA, CDCRC, Torrance, CA, USA. Email: mbudoff@lundquist.org. \\ Comment on: Singh T, Bing R, Dweck MR, van Beek EJR, Mills NL, Williams MC, Villines TC, Newby DE, Adamson PD. Exercise \\ Electrocardiography and Computed Tomography Coronary Angiography for Patients With Suspected Stable Angina Pectoris: A Post Hoc Analysis \\ of the Randomized SCOT-HEART Trial. JAMA Cardiol 2020. doi: 10.1001/jamacardio.2020.1567.
}

Submitted Jul 31, 2020. Accepted for publication Aug 14, 2020.

doi: 10.21037/qims-2020-24

View this article at: http://dx.doi.org/10.21037/qims-2020-24

Coronary heart disease (CHD), or coronary artery disease (CAD), is caused by the narrowing and hardening of arteries that supply blood to the heart, which occurs due to a buildup of plaque and cholesterol within the walls of the arteries. Atherosclerosis is the term that is used to describe this buildup within the coronary arteries (1). Progression of atherosclerosis can decrease the amount of oxygenrich blood that flows into the heart, and this can result in ischemia, injury, and/or infarct. Risk factors and causes of CAD include smoking, hypertension, high blood lowdensity lipoprotein (LDL) cholesterol levels, and diabetes mellitus. Other risk factors include age, male gender, family history, obesity, unhealthy diet, and large amounts of stress. CAD effects approximately 18.2 million adults aged 20 and above, and it is the most prevalent form of heart disease in the United States (2).

Evaluation of CAD is conducted by various modalities including exercise ECG and cardiac computed-tomography angiography (CTA). Limitations of exercise ECG testing include musculoskeletal conditions, left bundle branch blocks, health concerns/obesity, and diabetic neuropathy. All of these limitations could make it very difficult for a patient to complete an exercise ECG. CTAs have limitations that include allergies to contrast, renal disease, and radiation exposure. Overall, CTA has less limitations compared to exercise ECG.

Regarding this study by Singh et al., the authors focused on the diagnostic and prognostic abilities of exercise ECG and CTA. For individuals that underwent invasive coronary angiography, it was determined that exercise ECG has a sensitivity of $39 \%$ and a specificity of $91 \%$. CTA was found to have a sensitivity of $97 \%$ and a specificity of $86 \%$ for diagnosing and excluding CAD. A 97\% sensitivity means that the test is excellent at diagnosing and excluding disease (3).

In this well written, post-hoc analysis of the SCOTHEART trial 3,283 of 4,146 SCOT-HEART patients were randomized in a $1: 1$ ratio to either receive standard care or standard care plus CTA; 1,651 participants were designated to undergo coronary CTA. Standard care included a clinical evaluation and exercise ECG, if deemed necessary. Individuals that completed an exercise ECG used the Bruce Protocol. The goal time of exercise for patients was between $6-12$ minutes and $\geq 85 \%$ of their maximum predicted heart rate. The attending physician(s) determined the results of the exercise ECG(s) as either normal, inconclusive, or abnormal. CTA results were defined as stenosis with a luminal cross-sectional area as normal $(<10 \%)$, nonobstructive ( $\geq 10$ and $\leq 70 \%)$, or obstructive $(>70 \%)$. Prognoses, diagnoses, and treatments were determined by the attending clinician(s) and were not protocol-based. In addition, exercise ECG results were not adjudicated. After 6 weeks clinicians were asked to review their diagnoses, investigations, and treatments for both study groups. Changes were documented after review. 
It was discovered that $925(65 \%)$ of the patients that had normal results from the exercise ECG had either obstructive $(137,15 \%)$ or nonobstructive CAD $(379,41 \%)$ that was discovered on CTA. From the entire population, 137 (39\%) of 349 individuals that had obstructive CAD had exercise ECG results that were normal. For those patients with obstructive CAD, it was commonly identified by abnormal exercise ECG results. In those participants with a normal or inconclusive exercise ECG, the diagnosis of obstructive CAD was more frequently detected with the use of CTA $v s$ standard care alone [88 of 112 patients (79\%) vs. 47 of 197 patients (24\%)]. Referral for invasive coronary angiography was more frequent for individuals that had coronary CTA compared to participants designated to receive standard care alone, specifically for those that had exercise ECG results designated as inconclusive or normal. Abnormal or inconclusive results of exercise ECG were correlated with coronary revascularization at one year, as was nonobstructive and obstructive CAD that was detected on coronary CTA. Coronary revascularization was more frequently performed on patients who underwent coronary CTA.

At 5 years, those participants that had abnormal exercise ECG results had a greater incidence of death due to CAD or nonfatal myocardial infarction (MI) compared to individuals who had normal or inconclusive results. Surprisingly, the greater number of cardiac events occurred in those who had normal or inconclusive exercise ECG results (60 of 90 events, $66.7 \%$ ). Death from CAD or nonfatal MI was positively correlated with participants who had a presence of obstructive or nonobstructive CAD. Individuals who underwent coronary CTA had fewer cardiac events, compared to those who underwent exercise ECG alone and had abnormal or inconclusive results (19 of 549 patients, $3 \%$ vs. 31 of 549 patients, $6 \%$ ).

In this study, it was found that participants with abnormal exercise ECG results had a strong indication of obstructive CAD and a greater risk of $\mathrm{MI}$ and/or a need for future coronary revascularization. It is worrisome that a large number of individuals who had exercise ECG results determined as normal or inconclusive had nonobstructive or obstructive CAD that was not detected. Opposed to exercise ECG, coronary CTA was correlated with a stronger association of 5 -year CHD death or nonfatal MI ( $\mathrm{P}=0.002)$. Coronary CTA was also determined to be a more sufficient diagnostic tool for those patients who had normal or inconclusive exercise ECG results.

In 2015, results of the PROMISE trial were published.
In this trial, 10,003 symptomatic patients were randomly assigned to initial anatomical testing with CTA vs. functional testing (exercise ECG, nuclear stress testing, or stress echocardiography). The composite primary endpoint of death, MI, hospitalization for unstable angina, or major procedural complication was similar in the study groups. However, at 12 months, the risk of death or nonfatal MI was significantly lower in the CTA group $(\mathrm{P}=0.049)$ (4). Results of the SCOT-HEART trial were published in 2015 and 2018, correlated with 2- and 5-year outcomes, respectively. In this trial, 4,146 participants with suspected angina due to CAD were randomly assigned to standard care plus CTA vs. standard care alone. At 2-year follow up, compared to standard care alone, CTA significantly reclassified the diagnosis of $\mathrm{CAD}$ and the diagnosis of angina due to $\mathrm{CAD}$ $(\mathrm{P}<0.0001)$. In the CTA group, planned investigations and treatments were significantly altered compared to the standard care alone group. After 1.7 years, the use of CTA was associated with a $38 \%$ reduction in fatal and nonfatal MI, which barely missed the cutoff for statistical significance $(\mathrm{P}=0.0527)$. At 5 -year follow up, participants assigned to CTA were significantly more likely to be started on preventative and anti-anginal therapies. There was no difference between the groups in the frequency of invasive coronary angiography. However, the rate of the primary long-term endpoint (death from $\mathrm{CAD}$ or nonfatal $\mathrm{MI}$ ) was significantly lower in the CTA group $v s$. the standard care group $(\mathrm{P}=0.004)(5)$.

The question remains whether exercise ECG should remain the primary noninvasive diagnostic tool used to assess the presence of CAD. Cardiovascular symptoms that could indicate CAD are angina, shortness of breath, weakness, nausea and light-headedness. The SCOTHEART trial demonstrated that CTA is a viable tool for diagnosing CAD in patients that have stable angina, and CTA significantly resulted in better clinical outcomes than standard-care alone $(\mathrm{P}=0.004)(5)$. The rate of death from CAD or nonfatal MI was lower in the CTA group $(2.3 \%)$ than in the standard-care group $(3.9 \%)$. Also, in the PROMISE trail CTA was determined to better predict future events compared to functional testing (4). In the 5 -year analysis SCOT-HEART trial it was concluded that CTA increased the certainty and frequency of the diagnosis of CAD (5). In both the SCOT-HEART and PROMISE trials it was discovered that the use of CTA resulted in higher rates of detection of obstructive CAD than standard care or functional testing alone $(4,5)$.

A key point from this study by Singh et al., is the very 
low sensitivity of $39 \%$ for exercise ECG, which means that $61 \%$ of results are false negatives. This leaves those patients with no treatment for their disease, which may result in a serious cardiovascular event or death if CAD is not diagnosed. The true utility of any diagnostic test should be its able to positively influence outcomes. The true power of CTA is not its ability to direct patients to the catheterization lab but rather its ability to influence clinician's decision to initiate preventative therapies. In the PROMISE and SCOT-HEART trials both the diagnosis and therapeutic interventions were superior in the CTA groups $(4,5)$. In particular, the use of statin therapy and aspirin were significantly higher in the CTA groups.

Per this post hoc analysis of the SCOT-HEART trial, $65 \%$ of the participants $(n=925)$ that had normal exercise ECG test results, obstructive CAD was detected in 15\% of those individuals $(\mathrm{n}=137)$ and nonobstructive $\mathrm{CAD}$ was detected in $41 \%(n=379)$ on CTA. The significance of this is that those $65 \%$ of patients may not have had a secondary CTA exam in a regular clinician's office. That leaves a large population of patients who could be severely at risk of suffering a cardiovascular event or death due to undetected nonobstructive or obstructive CAD. Due to the limitations of exercise ECG, cardiac CTA should become the primary noninvasive diagnostic tool for detecting $\mathrm{CAD}$. For patients with suspected CAD, the primary diagnostic modality should be one that utilizes anatomical imaging. Further, CTA can define the location and extent of disease severity.

For CTA there is a very high sensitivity (97\%) and specificity $(86 \%)$, both indicating that the results are accurate and reliable for a physician to make a diagnosis from. Rather than risk a patient's life with an exercise ECG that may give a false negative and could risk their health, CTAs have a low incidence of both false negatives and false positives. While the specificity of CTA is $86 \%$, this means $14 \%$ of results will be false positives. Furthermore, we believe that exercise ECG should be reserved for equivocal results. As stated in the SCOT-HEART trial, for patients who received a correct diagnosis of CAD via CTA, invasive coronary angiography and coronary revascularization were used more appropriately. When patients receive the correct diagnosis, it is more likely that those patients will be treated with the correct preventative therapies and may have healthier lifestyle modifications implemented (5). The key to a correct diagnosis is using the proper diagnostic tools, and we believe that using CTA as the primary noninvasive exam for patients suffering from stable angina will reduce the risk of those patients suffering from future cardiovascular events and will improve patient outcomes.

Truly, seeing is believing.

\section{Acknowledgments}

Funding: None.

\section{Footnote}

Provenance and Peer Review: This article was commissioned by the editorial office, Quantitative Imaging in Medicine and Surgery. The article did not undergo external peer review.

Conflicts of Interest: Both authors have completed the ICMJE uniform disclosure form (available at http://dx.doi. org/10.21037/qims-2020-24). The authors have no conflicts of interest to declare.

Open Access Statement: This is an Open Access article distributed in accordance with the Creative Commons Attribution-NonCommercial-NoDerivs 4.0 International License (CC BY-NC-ND 4.0), which permits the noncommercial replication and distribution of the article with the strict proviso that no changes or edits are made and the original work is properly cited (including links to both the formal publication through the relevant DOI and the license). See: https://creativecommons.org/licenses/by-nc-nd/4.0/.

\section{References}

1. Coronary Artery Disease. Centers for Disease Control and Prevention. 2020 [cited 10 July 2020]. Available online: https://www.cdc.gov/heartdisease/coronary_ad.htm

2. Heart Disease Facts. Centers for Disease Control and Prevention. 2020 [accessed 10 July 2020]. Available online: https://www.cdc.gov/heartdisease/facts.htm

3. Singh T, Bing R, Dweck MR, van Beek EJR, Mills NL, Williams MC, Villines TC, Newby DE, Adamson PD. Exercise Electrocardiography and Computed Tomography Coronary Angiography for Patients With Suspected Stable Angina Pectoris: A Post Hoc Analysis of the Randomized SCOT-HEART Trial. JAMA Cardiol 2020;5:1-10.

4. Douglas PS, Hoffmann U, Patel MR, Mark DB, AlKhalidi HR, Cavanaugh B, Cole J, Dolor RJ, Fordyce CB, Huang M, Khan MA, Kosinski AS, Krucoff MW, Malhotra V, Picard MH, Udelson JE, Velazquez EJ, Yow E, Cooper LS, Lee KL; PROMISE Investigators. Outcomes of anatomical versus functional testing for coronary artery 
disease. N Engl J Med 2015;372:1291-300.

5. SCOT-HEART Investigators, Newby DE, Adamson PD, Berry C, Boon NA, Dweck MR, Flather M, Forbes J, Hunter A, Lewis S, MacLean S, Mills NL, Norrie J, Roditi
G, Shah ASV, Timmis AD, van Beek EJR, Williams MC. Coronary CT Angiography and 5-Year Risk of Myocardial Infarction. N Engl J Med 2018;379:924-33.

Cite this article as: Mikielski K, Budoff $M$. To stress or not to stress: a new approach for diagnosing coronary heart disease. Quant Imaging Med Surg 2020;10(12):2392-2395. doi: 10.21037/qims-2020-24 